\title{
A pancreatic hemorrhagic pseudocyst with pseudoaneurysm and the role of doppler ultrasonography: a case report
}

Kyunghwa Ryu ${ }^{1}$

(iD) Seona Sook Hong ${ }^{1}$

(iD) Hwajin Cha ${ }^{1}$

iD jiyoung Hwang

(iD) Eunfi Lee

Young Deok Cho

iD Yun-Woo Chang 1

(iD) Yong Jae Kim ${ }^{1}$

1. Department of Radiology, Soonchunhyang University Seoul Hospital, Yongsan-gu, Seoul, Korea 2. Digestive Disease Center and Research Institute, Department of Internal Medicine, Soonchunhyang University Seoul Hospital, Yongsan-gu, Seoul, Korea

http://dx.doi.org/10.1590/1806-9282.65.2.123

\section{SUMMARY}

Hemorrhagic pseudocysts with pseudoaneurysms are a rare and fatal complication of chronic pancreatitis due to the erosion of pancreatic to peripancreatic arteries. The timing of the rupture cannot be accurately predicted, but prompt diagnosis and management are essential to prevent further bleeding.

We describe the case of a 68-year-old man who presented acute epigastric pain and anemia and had a history of chronic pancreatitis with a pseudocyst. A biliary and pancreas MRI showed an enlarged size of a known pancreatic pseudocyst with internal high signal intensity material. Color-Doppler ultrasonography showed pulsating signals in the pseudocyst, and our final diagnosis was a pseudoaneurysm in the pancreatic hemorrhagic pseudocyst. The pseudoaneurysm was successfully treated with coil embolization of the feeding artery.

We report this case of a rare complication of chronic pancreatitis to show that color-Doppler ultrasound is a non-invasive and effective diagnostic tool for pseudoaneurysm, which enables early detection and prompt treatment without the need for invasive diagnostic modalities.

KEYWORDS: Pancreas. Aneurysm, false/diagnostic imaging. Pancreatic pseudocyst. Embolization, therapeutic.

\section{INTRODUCTION}

Hemorrhagic pseudocysts with pseudoaneurysms are a rare and fatal complication of chronic pancreatitis'. The following three mechanisms account for pseudoaneurysms related to pancreatitis: 1) severe inflammation and enzymatic autodigestion of a pancreatic or peripancreatic artery producing arterial disruption with pseudoaneurysm formation; 2) an established pseudocyst eroding into a visceral artery, thereby converting the pseudocyst into a large pseudoaneurysm; and 3) a pseudocyst eroding the bowel wall with bleeding from the mucosal surface ${ }^{2}$. It has been reported that a pseudoaneurysm following pan- 
creatitis occurs most commonly at the splenic artery, followed by the gastroduodenal, pancreaticoduodenal, left gastric, and common hepatic arteries ${ }^{3}$. Mortality rates due to bleeding pseudoaneurysms can be up to $40 \%$ depending on patient clinical status, site, and characteristics of the bleeding lesion and surgical procedure employed ${ }^{2}$. The timing of rupture is unpredictable; therefore early diagnosis and correct management are essential in preventing the fatal outcomes ${ }^{4}$. In our case, the patient had a pancreatic hemorrhagic pseudocyst with pseudoaneurysm and color Doppler imaging could speed the diagnosis with prompt management.

\section{CASE}

A 68-year-old man visited our outpatient clinic for epigastric pain without other gastrointestinal symptoms such as vomiting, diarrhea, or GI tract bleeding. The patient had been diagnosed with chronic pancreatitis with a pseudocyst 2 years ago outside the hospital. Also, he was diagnosed with portal vein thrombosis and treated with Warfarin for a month. At the time of admission, the patient's blood pressure was 110/70mmHg. Hemoglobin ( $\mathrm{Hb})$ was decreased by $9.0 \mathrm{~g} / \mathrm{dL}$, and hematocrit (Hct) was 29.3\%. Prothrombin time (PT) was prolonged to $36.3 \mathrm{sec}$ and activated partial thromboplastin time (aPTT) was also prolonged to $49.9 \mathrm{sec}$

Biliary and pancreas dynamic T1-weighted axial MR image revealed an enlarged known pseudocyst in the pancreas head filled with high signal intensity contrast material in the lesion (Fig.1). Also, pancreas showed diffuse parenchymal swelling with peripancreatic fat infiltration.

Ultrasonography was performed for further characterization of the lesion. Grayscale ultrasonography showed about a heterogeneously hyperechoic lesion of approximately $4 \mathrm{~cm}$ at the pancreas head, which correlated with hemorrhagic pseudocyst on previous biliary and pancreas MRI. There was a hypoechoic sac of about $1.3 \mathrm{~cm}$ located at the anteroinferior portion of the pseudocyst. In the sac, Color Doppler ultrasonography showed a typical swirling signal called "yin-yang" sign and arterial flow was detected on duplex Doppler ultrasonography (Fig. 2A \& 2B). Based on the findings of the color Doppler imaging, pseudoaneurysm in pancreatic pseudocyst was considered. The physician decided to perform embolization for pseudoaneurysm treatment. A selective

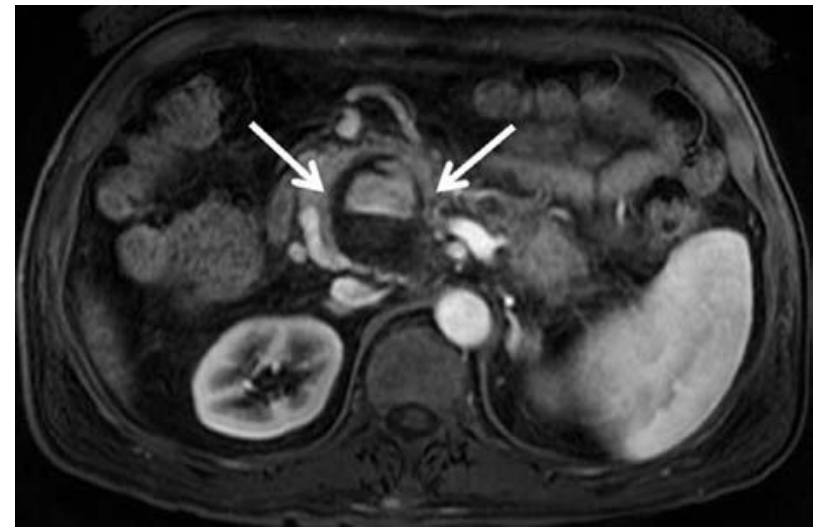

FIGURE 1. Biliary and pancreas dynamic T1-weighted axial MR image shows a contrast-filled pseudocyst of about $4.4 \times 3.8 \mathrm{~cm}$ at the pancreas head portion, which is suspected to be a hemorrhagic pseudocyst (arrows).
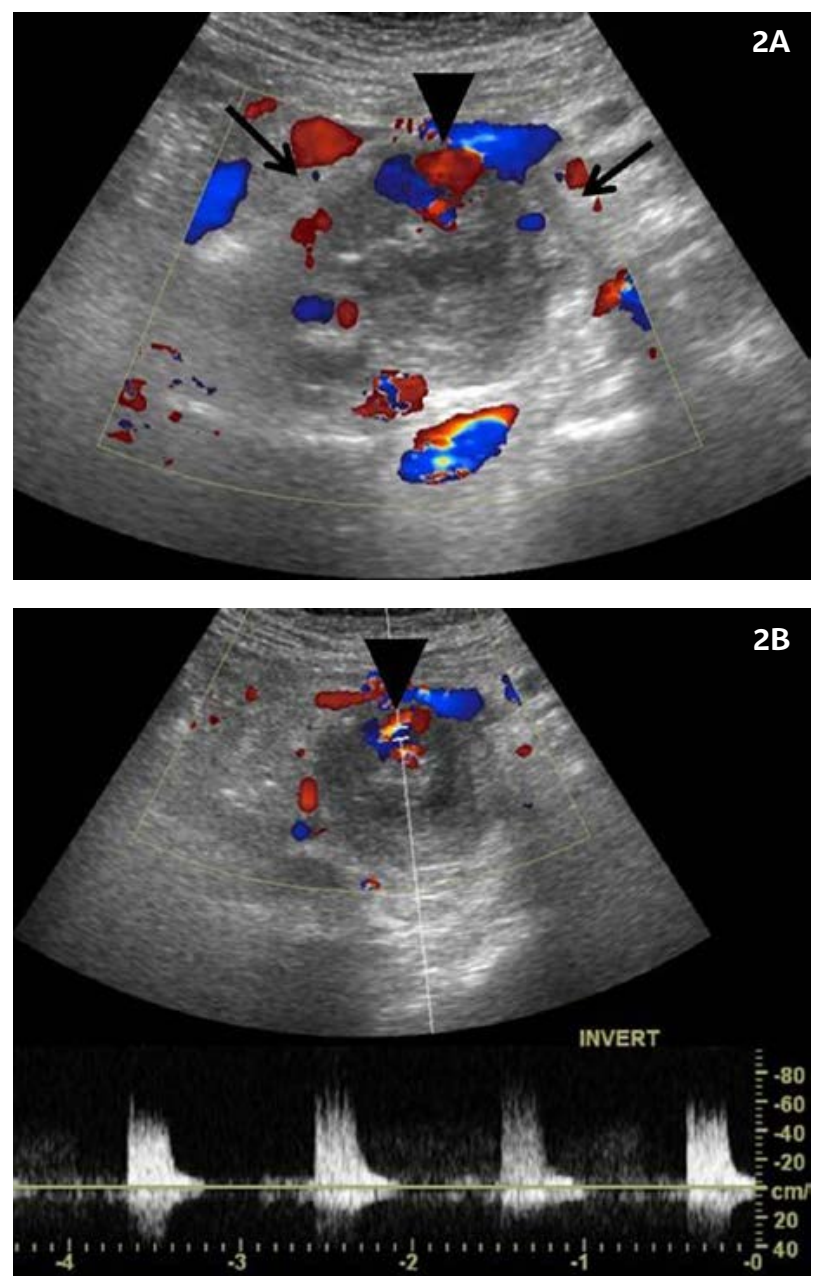

FIGURE 2A. Color Doppler ultrasonography shows a heterogeneously hyperechoic pseudocyst of about $4 \mathrm{~cm}$ at the pancreas head and an anechoic sac located at the anterior portion of the pseudocyst wall. The typical swirling signal called "yin-yang" sign is seen in the sac (arrowhead).

FIGURE 2B. Duplex Doppler ultrasonography depicts prominent arterial flow in the sac. 


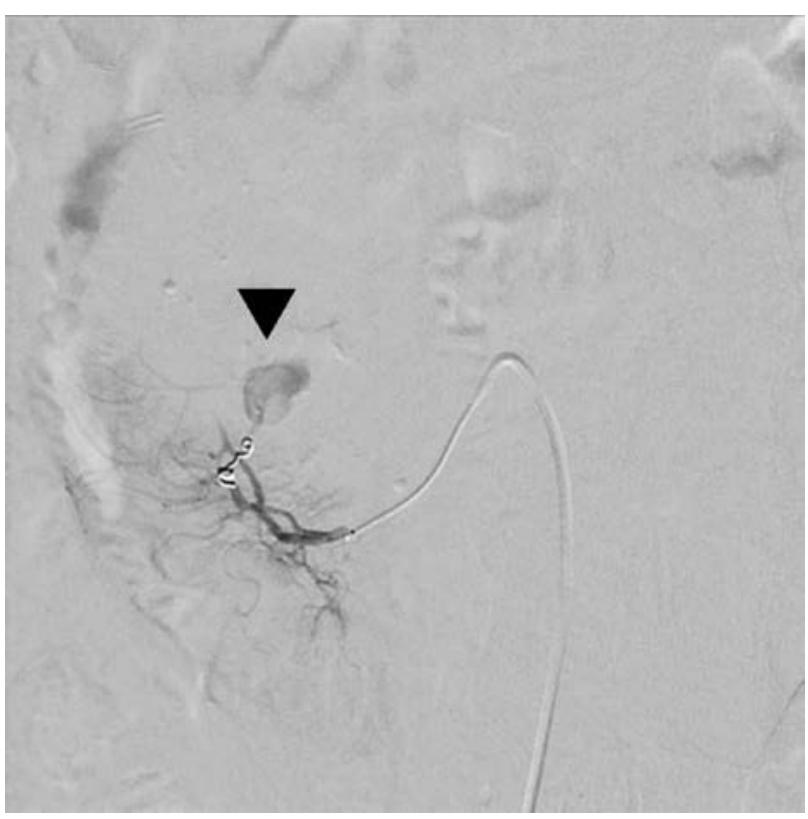

FIGURE 3. Digital subtraction angiogram shows the pseudoaneurysm (arrowhead) during coil embolization of pancreaticoduodenal artery of superior mesenteric artery.

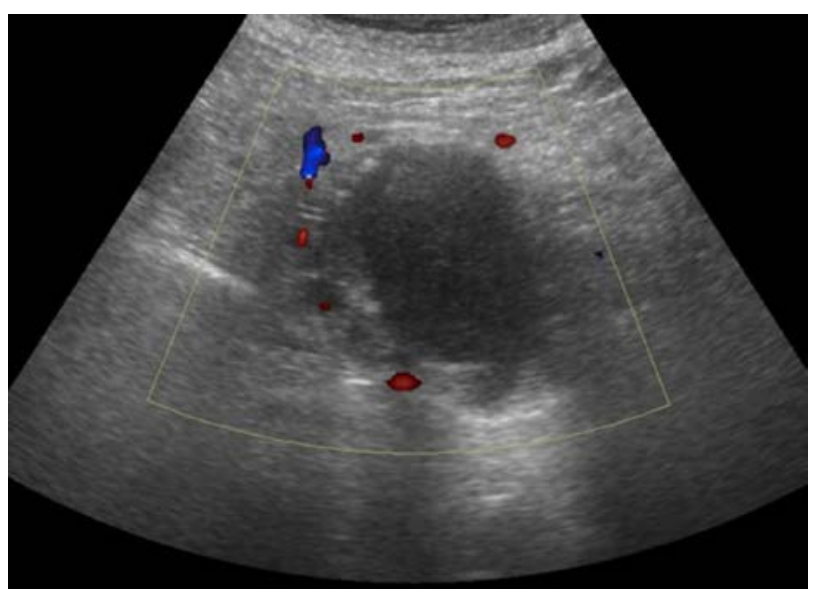

FIGURE 4. Follow-up color Doppler ultrasonography 6 days after the interventional treatment shows no more vascularity within the pseudocyst.

angiogram of the superior mesentery artery showed the pseudoaneurysm was being fed by the $1^{\text {st }}$ branch of the superior mesenteric artery. Coil was deployed and on post coil embolization angiogram, the pseudocyst was not excluded on arterial flow. Besides the first branch of the superior mesenteric artery, other feeding vessels were suspected. a glue-lipiodol mixture was injected into the pseudoaneurysm and control angiography confirmed total exclusion of the pseudoaneurysm (Fig.3). 6 Days later, follow up abdominal ultrasonography, and color Doppler imaging was performed, and vascularity was no longer seen in the pseudocyst (Fig. 4).

\section{DISCUSSION}

Hemorrhage is uncommon in pancreatic pseudocysts, but it is a potentially life-threatening complication. According to the literature, the incidence of pancreatic pseudoaneurysm in patients with chronic pancreatitis is less than $10 \%$, and spontaneous hemorrhage arising from a pancreatic pseudocyst reportedly ranges from 1.4 to $8.4 \%$. When bleeding occurs, it can be potentially lethal, as mortality rates can reach up to $40 \%{ }^{5}$. Therefore early detection of pseudoaneurysm and appropriate treatment is required in preventing massive bleedings. The combination of angiographic embolization and surgery is thought to be the most appropriate management.

The diagnosis of bleeding into a pancreatic pseudocyst should be suspected in the presence of any of the following conditions: (1) sudden enlargement of previously present pseudocyst; (2) development of a bruit over a pseudocyst in a patient with or without abdominal pain; (3) upper gastrointestinal bleeding in a patient with a known pseudocyst and no other source seen on upper gastrointestinal endoscopy; or (4) a sudden decrease in hematocrit with no evidence of gastrointestinal bleeding in a patient with a pancreatic pseudocyst ${ }^{6}$.

The diagnostic modalities of hemorrhagic pseudocyst include contrast-enhanced CT scan, ultrasonography, and angiography. On CT scan, pseudoaneurysms are readily diagnosed as rapidly enhancing intracystic lesions, with attenuations similar to those of the aorta and other larger splanchnic arteries. Ultrasonography is a valuable tool for diagnosis of pseudoaneurysms, and it has been widely utilized as a noninvasive imaging modality for investigation of vascular disease. The gray-scale US illustrates pseudoaneurysm as a hypoechoic cystic structure nearby a supplying artery; however, its findings are accompanied by other clinical conditions such as hematomas and cystic masses. Therefore color Doppler US can be used for confirmation for pseudoaneurysm ${ }^{7}$. Blood flow within a cystic structure is characterized by a typical swirling motion called the "yin-yang sign," however this pattern of flow can also be seen in a saccular aneurysm, so diagnosis made from this finding alone may prove to be inaccurate. The hallmark of the diagnosis is the demonstration of a communicating channel between the sac and the feeding artery with a "to-and-fro" waveform at the duplex Doppler US. Conventional angiography remains the 
standard reference for diagnosis but it is an invasive procedure, and noninvasive diagnostic modalities should be included in the initial work-up if possible ${ }^{8}$.

In our case, color Doppler ultrasonography allowed a quick diagnosis of pancreatic hemorrhagic pseudocyst with pseudoaneurysm and prompt treatment before massive hemorrhage could occur. Post-embolization doppler imaging also showed a change in vascularity within the pseudocyst, which confirmed the complete exclusion of the pseudoaneurysm. Therefore, Doppler ultrasonography is a safe and effective diagnostic tool and can be useful to follow up pseudoaneurysms without the need for invasive diagnostic modalities.

\section{Acknowledgments}

This work was supported in part by the Soonchyunhyang University Research Fund.”

PALAVRAS-ChaVe: Pâncreas. Falso aneurisma/diagnóstico por imagem. Pseudocisto pancreático. Embolização terapêutica.

\section{REFERENCES}

1. von Flüe M, Kocher T, Herzog U, Looser C, Schuppisser JP. Hemorrhage from pseudocysts caused by pseudoaneurysms in chronic pancreatitis. Diagnosis and management. Helv Chir Acta. 1993;59(5-6):785-9.

2. Hsu JT, Yeh CN, Hung CF, Chen HM, Hwang TL, Jan YY, et al. Management and outcome of bleeding pseudoaneurysm associated with chronic pancreatitis. BMC Gastroenterol. 2006;6:3.

3. Masatsugu T, Yamaguchi K, Yokohata K, Mizumoto K, Chijiiwa K, Tanaka M. Hemorrhagic pseudocyst and pseudocyst with pseudoaneurysm successfully treated by pancreatectomy: report of three cases. J Hepatobiliary Pancreat Surg. 2000;7(4):432-7.

4. Mitchell DG, Needleman L, Bezzi M, Goldberg BB, Kurtz AB, Pennell RG, et al. Femoral artery pseudoaneurysm: diagnosis with conventional duplex and color Doppler US. Radiology. 1987;165(3):687-90.
5. Novacic K, Vidjak V, Suknaic S, Skopljanac A. Embolization of a large pancreatic pseudoaneurysm converted from pseudocyst (hemorrhagic pseudocyst). JOP. 2008;9(3):317-21.

6. Kiviluoto T, Kivisaari L, Kivilaakso E, Lempinen M. Pseudocysts in chronic pancreatitis: surgical results in 102 consecutive patients. Arch Surg. 1989;124(2):240-3.

7. Mahmoud MZ, Al-Saadi M, Abuderman A, Alzimami KS, Alkhorayef M, Almagli $B$, et al. "To-and-fro" waveform in the diagnosis of arterial pseudoaneurysms. World J Radiol. 2015;7(5):89-99.

8. Saad NE, Saad WE, Davies MG, Waldman DL, Fultz PI, Rubens D). Pseudoaneurysms and the role of minimally invasive techniques in their management. Radiographics. 2005;25(Suppl 1):S173-89. 